\title{
Läßt sich in der Rückenmarksflüssigkeit eine für Lues spezi- fische Eiweißreaktion nachweisen?
}

\author{
Von \\ Priv.-Doz. Dr. V. Kafka. \\ (Aus dem serologischen Laboratorium der Staatskrankenanstalt und psychiatrischen \\ Universitätsklinik Friedrichsberg in Ha mburg.)
}

I.

Die Forschung der letzten Jahre hat sich intensiv mit dem Problem der durch die Syphilis gesetzten Veränderungen des Blutserums beschäftigt. Nachdem die Ergebnisse der WaR. bewiesen hatten, daß solche tatsächlich vorhanden und durch eine biologische Versuchsanordnung nachweisbar sind, war man bemüht, insbesondere mit Hilfe chemischer, biochemischer und kolloidchemischer Methodik die Eigenart des Syphilitikerserums klarzustellen, wobei man meist von der WaR. ąusging und neue Resultate wieder zur Erklärung der WaR. heranzog. Bei der Bearbeitung dieses Gebietes wurden eine Reihe praktisch wertvoller neuer Serumreaktionen gefunden; ich erwähne hier nur die Gerinnungsreaktion von Hirschfeld und Klinger, dann die Flockungsreaktionen von Sachs und Georgi, sowie Meinicke. Die Erkenntnis freilich der allen diesen Reaktionen zugrunde liegenden, für die Lues charakteristischen Phänomene zog trotz Auffindung einer großen Reihe wertvoller Einzelheiten nicht den entsprechenden Gewinn. Nachdem die Untersuchungen über quantitative Veränderungen der Serumeiweißkörper, zumal der verschiedenen Globuline, bei der Lues zu einem eindeutigen Ergebnis nicht geführt hatten, schien es auf Grund der Arbeiten von H. Sachs, U. Friedema n n, Bruck, Hirschfeld und Klinger, Meinicke u. a. am wahrscheinlichsten, eine ph ysi kalische Zustandsänderung der Serumglobuline als für Syphilis charakteristisch anzusehen und diese zugleich als Ursache der WaR., sowie der anderen praktisch brauchbaren Seroreaktionen bei Lues anzunehmen. Von Interesse ist von diesem Standpunkte aus eine neve Arbeit xon Gloor und Klinger, die gefunden haben, daß syphilitische Sera auch dann noch ausgesprochen positiv reagieren, wenn sie ihrer Globuline beraubt sind. Dieses Faktum scheint zumindest geeignet, die Veränderungen des Syphilitikerbluts nicht nur bei den Globulinen zu suchen. 
Benulzt Jnan, wie es die meisten Autoren getan haben, die WaR. als Ausgangspunkt für solche Untersuchungen, so muß es wundernehmen, daß bisher die Rückenmarksflüssigkeit fast gar nicht nach dieser Richtung bearbeitet worden ist. Können doch bei einer anscheinend gleich starken WaR. in Blut und Liquor, z. B. in einem Fall von Paralyse, große quantitative und qualitative Verschiedenheiten der Globuline der beiden Flüssigkeiten vorhanden sein. Wir haben nun, um diese Lücke einigermaßen auszufüllen, eine größere Reihe von Versuchen unternommen. Bevor diese mitgeteilt werden, sei kurz zusammengestellt, wie weit wir bisher für Lues spezifische Liquorreaktionen kennen.

Von der Phase I wurde bekanntlich anfänglich angenommen, daß sie nur bei Lues des Zentralnervensystems positiv sei, nicht aber bei Lues - ohne syphilitische Erkrankung des Z. N. S. und nicht bei nichtsyphilitischen organischen Erkrankungen des $Z$. N. S. Es stellte sich aber bald herans, daß hier nur graduelle Unterschiede vorliegen, daß also die Phase I auch bei nichtsyphilitischen organischen Erkrankungen des Z. N. S. positiv sein kann und die Forschung der letzten Jahre hat uns gelehrt, daß auch die Lues ohne klinische Erscheinungen von seiten des Z. N. S. mit einer positiven Phase I einhergehen kann.

Ein Gleiches gilt cum grano salis für dis durch die Reaktion nach Pand y und die Buttersäureprobe nach Noguchi gefällten Eiweißkörper.

Gegen alle 3 Reaktionen könnte man nun einwenden, daß, da sie ja im wesentlichen die Fällung aller Globuline bewirken, das Vorkommen eines eventuell für Lues spezifischen Globulins verschleiert wird. In Konsequenz dieses Gedankens hat Kafka analog ähnlichen, mit dem Blutserum angestellten Untersuchungen die verschiedenen Globuline durch fraktionierte Ammoniumsulfataussalzung zu trennen versucht. Dabei ergab sich, daß die nichtsyphilitischen organischen. Erkrankungen meist nur eine positive Phase I ergaben, die syphilitischen aber noch bei der 40 proz. Konzentration positive Befunde zeigen, wobei wieder die Paralyse und selten die Tabes auch bei der 33 proz. Konzentration meist noch reagierten, frische Gehirnlues mit schweren entzündlichen Liquorveränderungen zeigte sogar oft noch eine positive Reaktion bei der 28 proz. Konzentration. Wenn nun diese Reaktionen die diagnostische Bedeutung der Phase I erweitern halfen, so konnte doch keiner von ihnen Spezifität für Lues des Z. N. S. - nicht einmal klinische - zugebilligt werden, da alle Reaktionen, von der 28 proz. angefangen, bei der infektiösen nicht syphilitischen Meningitis mit negativer WaR. im Liquor positiv sein können.

Eine neuere von Weichbrodt eingeführte Reaktion, die Sublimatreaktion, schien eine größere Spezifität für Lues des Z. N. S. zu haben. Fand Weichbrodt sie doch in 3 Fällen von infektiöser Meningitis negativ, bei stark positiver Phase $\mathrm{I}$. Freilich gibt Weichbrodt in der gleichen Arbeit an, daß auch bei organischen Gehirnerkrankungen ohne Lues eine positive Sublimatreaktion vorhanden sein kann, daß aber die Trübungen der Rückenmarksflüssigkeiten der durch Lues bedingten Erkrankungen des Z. N. S. einen bläulichen Schimmer aufweisen. Marx konnte in einer Arbeit aus meinem Laboratorium diese Färbung nicht bestätigen; es zeigte sich zwar, daß sich das Reaktionsgebiet der Sublimatreaktion mit jenem der Phase I nicht völlig deckt, daß aber eine Spezifität für Lues dieser neuen Reaktion nicht eignet.

Auch für die Braun-Huslersche Reaktion kann eine Luesspezifität nicht ausgesprochen werden, da sie bei akuten, nichtsyphilitischen Meningitiden ein 
deutlich positives Ergebnis liefert. Das gleiche gilt für die verschiedenen Methoden zur Bestimmung des Gesamteiweißes; wir können daher zusammenfassen, daß die bisherigen chemischen Liquorreaktionen in ihrem qualita. tiven Ausfall fürLues nicht spezifisch sind, da $B$ nur das quantitative Resultat uns für die verschiedenen Luesformen charakteristische Ergebnisse liefern kann.

Einen wesentlichen Fortschritt schienen hier die Kolloidreaktionen zu bedeuten, die ja für die verschiedenen Formen der Lues des Zentralnervensystems charakteristische Kolloidveränderungskurven bieten. Wir können aber heute nur sagen, daß die verschiedenen Kurvenformen (Paralysen-, Lues cerebri- Kurve, Lueszacke) uns für die Luesdiagnose sehr verwendbar Anhaltspunkte liefern, daß aber auch hier eine Luesspezifität bisher nicht nachzuweisen ist, was schon aus der Verschiedenheit der Kurvenformen in den verschiedenen Luesstadien hervorgeht, sowie dem Vorkommen ähnlicher Kurventypen bei nicht syphilitischen organischen Erkrankungen des Z. N. S.

\section{II.}

Unsere eigenen Versuche mußten sich auf die Untersuchungen stützen, die nach dieser Richtung hin mit dem Blutserum vorgenommen worden waren. Dabei muß eine Schwierigkeit der Bearbeitung vorausgeschickt werden. Während sich die Blutsera Syphilitischer und Nichtsyphilitischer leicht vergleichen lassen, da ihr Aufbau an Eiweißkörpern speziell Globulinen sehr ähnlich ist, wissen wir, daß der normale Liquor Globuline nur in Spuren enthält. Es war daher zur Beantwortung einiger Fragestellungen nötig, daß die Ergebnisse des pathologischen Liquors mit Globulinvermehrung und positiver WaR. mit jenen des pathologischen und normalen Serums einerseits, andererseits mit jenen eines pathologischen Liquors mit Globulinvermehrung und negativer WaR. (Fälle, die nicht zu häufig sind, wenn wir von der akuten infektiösen nichtsyphilitischen Meningitis absehen) zu vergleichen und vom normalen Liquor: im Prinzip abzusehen.

Vor allem interessierte uns die Frage, wie weit die von U. Friedemann für das Blutserum gefundenen Frgebnisse für die Rückenmarksflüssigkeit Gültigkeit haben. Dieser Autor hatte nämlich festgestellt, daß auch die Globuline normaler Sera die WaR. geben, daß diese aber nicht in Erscheinung tritt, weil die Albumine eine hemmende Wirkung ausüben. Im Syphilitikerserum findet nun diese antagonistische Wirkung der Albumine nicht statt, so daß die WaR. zutage tritt. Die Ursache ist in einer Veränderung der Globuline des Sy philitikerserums zu suchen.

In der Technik der Versuche haben wir uns eng jener von U. Friede mann angeschlossen; sie mußte aber an vielen Punkten verändert werden, da die Globuline wassermannpositiver Rüickenmarksflüssigkeiten ja in viel geringerer Menge vorhanden sind, als die der Sera und auch qualitativ sich mit jenen nicht decken.

Unsere Versuche, über die a. a. 0 . ausführlich zu berichten sein wird, haben nun ergeben, daß sich die Globuline wassermann- 
positiver Rückenmarksflüssigkeiten ebenso verhalten, wie jene der a ktiven Sera, d. h. sie geben eine stark positive WaR. ohne Selbsthemmung. Die Globuline wassermann negativer Rückenmarksflüssigkeiten zeigten meist negative, selten schwach positive WaR.; es zeigte sich hier ein geringer Unterschied gegenüber der Wirkung der Globuline wassermannnegativer aktiver Sera, der vielleicht in der geringen Menge der normalen Liquorglobuline begründet ist. Bezüglich der isolierten Albumine fand sich folgendes Verhältnis:

Tabelle I.

Albumine des

\begin{tabular}{c|c|c|c}
\hline $\begin{array}{c}\text { WaR.-positiven } \\
\text { Liquors }\end{array}$ & $\begin{array}{c}\text { WaR.-negativen } \\
\text { Liquors }\end{array}$ & $\begin{array}{c}\text { WaR.-positiven } \\
\text { aktiven Serums }\end{array}$ & $\begin{array}{c}\text { WaR.-negativen } \\
\text { aktiven Serums }\end{array}$ \\
\hline $\begin{array}{c}\text { negativ oder Hem- } \\
\text { mung mit leichter } \\
\text { Selbsthemmung }\end{array}$ & $\begin{array}{c}\text { starke } \\
\text { Selbsthemmung }\end{array}$ & Selbsthemmung & negativ. \\
\hline
\end{tabular}

Von Wichtigkeit sind nun die Kombinationsversuche, wie sie auch U. Friede mann ausgeführt hat. Ihre Ergebnisse sind am übersichtlichsten in einer Tabelle (II) dargestellt.

Tabelle II.

Kombinationsversuche.

\begin{tabular}{c|c}
\hline Kombination & Ergebnis \\
\hline Glob. pos. akt. Se. + Alb. pos. akt. Se. & pos. WaR. \\
Glob. pos. akt. Se. + Alb. neg. akt. Se. & pos. WaR. \\
Glob. neg. akt. Se. + Alb. pos. akt. Se. & neg. WaR. \\
Glob. neg. akt. Se. + Alb. neg. akt. Se. & neg. WaR. \\
Glob. pos. Liq. + Alb. pos. Liq. & Additionswirkung oder Hem- \\
mung mit Abschwächung. & Additionswirkung \\
Glob. pos. Liq. + Alb. neg. Liq. & Abschwächung der WaR. \\
Glob. pos. Liq. + Alb. neg. akt. Se. & ebenso \\
Glob. pos. Liq. + Alb. pos. akt. Se. &
\end{tabular}

Wir sehen also hier, trotzdem die WaR. des negativen Serums und Liquors ausgeweitet gleich erschien, doch deutliche Unterschiede in den Kombinationsversuchen, die darauf hindeuten, daß die Versuche von U. Friedemann eine Verallgemeinerung nicht vertragen und daß die Ergebnisse dieser Versuche zwar sehr interessant, für die Beurteilung der WaR. aber nicht ausschlaggebend sein können, daß vor allem die Globuline in Blut und Liquor sich trotz anscheinend gleich positiver WaR. der Gesamtflüssigkeit isoliert verschieden verhalten. Hierin unterstützen uns die Ergebnisse von Gloor und Klinger, die gefunden haben, daß die syphilitischen Sera auch nach Entfernung der Globuline (durch 
n/300 $\mathrm{HCl}$ ) positiv reagieren, ein Phänomen, daß die Autoren im globulinfreien Serum Affinitäten annehmen, die dadurch zustande kommen, „daß bei den durch die Spirochäten bedingten pathologischen Zellveränderungen gewisse Abbauprodukte auftreten ${ }^{(1)}$ ).

Wir können hier auf diese interessanten und recht komplizierten Verhältnisse nicht weiter eingehen, sondern müssen zu Versuchen übergehen, die sich mit der Frage des biologischen Nachweises obiger Abbauprodukte befassen. Bekanntlich haben Much und Emden auf Grund ihrer Versuche angenommen, daß die WaR. durch im Blute vorhandene Eiweißabbauprodukte hervorgerufen wird. Versuche von M. Fraenkel, der Sera gegen destilliertes Wasser dialysierte, und positive WaR. im Dialysat bei 1,0 erhielt und der diese Ergebnisse zur Stütze der Muchschen Anschauungen verwertete, konnte ich darauf zurückzuführen, daß die erhaltenen positiven Reaktionen des Dialysats nur Folge seiner Salzarmut sind, da das besalzene Dialysat negativ nach Wassermann reagiert. Auch die Veränderungen des Hülseninhalts in bezug auf Komplementbindung sind darauf zurückzuführen, da besalzenes dialysiertes Serum ebenso reagiert, wie das negative.

Da derartige Versuche mit dem Liquor noch nicht vorgenommen, sei einer von den vielen, die von uns ausgeführt worden sind, hier gebracht.

\section{Tabelle III.}

WaR. des dialysierten Liquors und der Dialysate.

\begin{tabular}{|c|c|c|c|c|c|c|c|}
\hline & \multirow{3}{*}{ Flüssigkeit } & \multicolumn{6}{|c|}{ WaR. } \\
\hline & & \multicolumn{3}{|c|}{ mit Extrakt } & \multicolumn{3}{|c|}{ obne Extrakt } \\
\hline & & 0,2 & 0,5 & 1,0 & 0,2 & 0,5 & 1,0 \\
\hline 1. & Liquor B. . . & & & $++t$ & & & $\theta$ \\
\hline 2. & Liquor Sch. . . . . & $\theta$ & $\theta$ & $\theta$ & & & $\theta$ \\
\hline 3. & Liquor B. $3 \mathrm{ccm}$ gegen $15 \mathrm{cem}$ dest. & & & & & & \\
\hline & Wasser, 16 St. dial. unbesalzen & $+t+$ & $1++$ & $t+t$ & & & $\theta$ \\
\hline 4. & $\begin{array}{r}\text { Liquor Sch. ebenso behandelt, } \\
\text { unbesalzen } . . . . . . . . . . .\end{array}$ & $\theta$ & $\theta$ & $+t$ & & & $\theta$ \\
\hline 5. & Dialysat von 3 unbesalzen. & $\theta$ & $\theta$ & $\theta$ & & & $\theta$ \\
\hline 6. & Dialysat von 4 unbesalzen. & $\theta$ & $\theta$ & $\theta$ & & & $\theta$ \\
\hline 7. & Dialysat von 3 besalzen. & $\theta$ & $\theta$ & $\theta$ & & & $\theta$ \\
\hline 8. & Dialysat von 4 besalzen . & $\theta$ & $\theta$ & $\theta$ & & & $\theta$ \\
\hline 9.1 & 3 besalzen . . . . . . . & $t+t$ & $++t$ & $t+t$ & & & $\theta$ \\
\hline 10. & 4 besalzen & $\theta$ & $\theta$ & $\theta$ & & & $\theta$ \\
\hline
\end{tabular}

Hierzu ist zu bemerken, daß die unbesalzenen Dialysate bei 2,0 meist positive WaR. geben, entsprechend der WaR. einer 0,3 bis 0,4 proz. NaCl-Lösung. Die positive WaR. des dialysierten negativen Liquors in unbesalzenem Zustand ist auf die Salzarmut und die dadurch

1) Zu der Arbeit von Gloor und Klinger ist freilich zu bemerken, daß durch $11 /$ soo $\mathrm{HCl}$ nur cin Teil der Globuline gefällt wird. 
hervorgerufenen Veränderungen zurückzuführen, da der besalzene dialysierte Liquor wieder negativ reagiert.

Es lassen sich also a uch a us dem Liquor nicht durch Dialyse Abbauprodukte gewinnen, die eine positive WaR. zu geben imstande sind.

Auch der chemische Nachweis von Eiweißabbauprodukten hat bisher keine für die Syphilis des Z.N.S. charakteristischen Ergebnisse gehabt. Die Ninhydrinreaktion des unbeeinflußten Liquors (Nobel) zeigt bei der akuten Meningitis die stärksten und bietet auch keine qualitativ verwertbaren Farbennuancen; die Ninhydrinreaktion des dialysierten Liquors (Kaf $\mathrm{ka}$ ) hat ebenfalls nach dieser Richtung hin zu befriedigenden Ergebnissen nicht geführt.

Wenden wir uns noch der Kolloidchemie zu, so haben wir, von dem Gedanken ausgehend, daß die sog. Kolloidreaktionen vor allem mit dem Indicator der Kolloidveränderung durch entgegengesetzte geladene Kolloide arbeiten und daß bei der gewöhnlichen Versuchsanordnung mit fallender Liquormenge der Salzgehalt steigt, die erhaltenen Kurven also rein empirische sind, versucht, reine Kolloidschutzkurven zu erhalten. Wir gingen dabei einem früheren Gedanken von C. Lange entsprechend so vor, daß wir absteigende Liquormengen mit destilliertem Wasser auf dasselbe Volumen verdünnten, dann die gleichen Mengen hochkonzentrierter Kochsalzlösungen (z. B. 10-15 proz.) hinzusetzten, hierauf die kolloidale Lösung (z. B. $5 \mathrm{ccm}$ einer Goldsol- oder I cem einer Mastixversuchslösung) eintrugen. Das Ergebnis eines solchen Versuches (Einzelheiten folgen a. a. O.) ergibt die folgende Kurve:

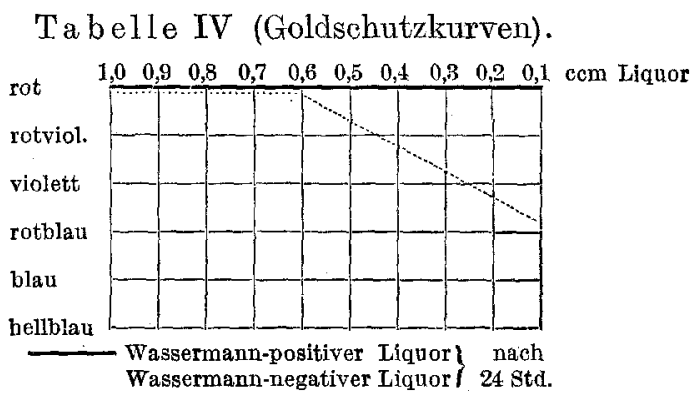

Es zeigt also der negativeLiquor einen deutlich geringeren Goldschutz als der positive. Interessant ist, daß auch bei $1,0 \mathrm{ccm}$ Liquor keine Veränderung des Kolloids eintritt. Eine solche ist bei Verwendung von Mastixlösungen nach mehreren Stunden zu beobachten, so daß man hier, wenn man Schutzkurve erhalten will, nach kurzer Zeit ablesen muß. Solche Kurven scheinen, wenn die Technilk des Versuches 
genau ausgeführt wird, uns noch viel Wichtiges für Liquordiag nostik vor allem quoad luem zu versprechen.

Schließlich haben wir Versuche gemacht, die physikalisehchemischen Verhältnisse der Liquorglobuline zu prüfen; so wurde doch den Serumglobulinen der Syphilitiker eine leichtere Fällbarkeit zugesprochen. Wir bedienten uns dabei' der Milchsäuremethode Brucks. Glücklicherweise stand uns neben wassermannpositiven und -negativen Rückenmarksflüssigkeiten eine solche einer Lues cerebri zur Verfügung, die bei negativer WaR. einen starken Globulingehalt hatte (Liquor II). Wir gingen also so vor, daß wir zu der gleichen Liquormenge $4 \mathrm{ccm}$ destilliertes Wasser und 1:1000 aufsteigende Milchsäuremengen hinzusetzten.

Tabelle V.

Milchsäureversuche.

\begin{tabular}{|c|c|c|c|c|c|}
\hline \multirow[b]{2}{*}{$\begin{array}{l}\text { Liquor } \\
\mathrm{ccm} \\
\end{array}$} & \multirow{2}{*}{$\begin{array}{c}\text { Aq. } \\
\text { dest. } \\
\text { com } \\
\end{array}$} & \multirow{2}{*}{$\begin{array}{c}\text { Mileh } \\
\text { säure } \\
1: 1000 \\
\text { ccm } \\
\end{array}$} & \multicolumn{3}{|c|}{ Ergebnis nach 10 Minuten } \\
\hline & & & $\begin{array}{c}\text { Liquor I } \\
\text { WaR. }++ \text { Ph.I }+++\end{array}$ & $\begin{array}{c}\text { Liquor II } \\
\text { WaR. } \theta \text { Ph. I + + }\end{array}$ & $\begin{array}{c}\text { Liquor III } \\
\text { WaR. } \theta \mathrm{Ph} . \text { I Opal. }\end{array}$ \\
\hline 0,2 & 4 & 0,05 & Opal. & Opal. & $\theta$ \\
\hline 0,2 & 4 & 0,1 & Opal. & Opal: & $\theta$ \\
\hline 0,2 & 4 & 0,2 & Opal. & Opal. & $\theta$ \\
\hline 0,2 & 4 & 0,3 & starke Opal. & starke Opal. & $\theta$ \\
\hline 0,2 & 4 . & 0,4 & deutI. Opal. & deutl. Opal. & $\theta$ \\
\hline 0,2 & 4 & 0,5 & Spur Opal. & Spur Opal. & $\theta$ \\
\hline 0,2 & 4 & 0,6 & $\theta$ & Spur Opal. & $\theta$ \\
\hline 0,2 & 4 & 0,7 & $\theta$ & $\theta$ & $\theta$ \\
\hline 0,2 & 4 & 0,8 & $\theta$ & $\theta$ & $\theta$ \\
\hline 0,2 & 4 & 0,9 & $\theta$ & $\theta$ & $\theta$ \\
\hline 0,2 & 4 & 1,0 & $\theta$ & $\theta$ & $\theta$ \\
\hline
\end{tabular}

Liquor I und II zeigen keine Unterschiede in der Fällbarkeit, bei beiden ist das Maximum am gleichen Punkte erreicht. Wir sind dann mit der Milchsäuremenge noch tiefer herabgegangen bis auf $0,01 \mathrm{ccm}$. Hier ergaben sich interessante Ergebnisse, insofern als der Eintritt der Milchsäurefällung einen gewissen Parallelismus mit der Stärke der WaR. zeigte. So beobachteten wir bei einem Liquor, bei dem die WaR. bei $0,2+++$ war, den Eintritt der Milchsäurefällung bei 0,04 , bei einem anderen, der eine positive WaR. erst bei 0,5 aufwies, den Eintritt der Milchsäurefällung bei 0,2. Freilich macht es den Eindruck, als ob auch dieser Teil der Milchsäurefällungskurve von der Quantität der Globuline abhinge. Immerhin scheinen derartige Versuche, die bei 0,0l Milchsäure I: 1000 anfangen, für die Beurteilung der physikalischen Beschaffenheit der Liquorglobuline nicht unwichtig zu sein. Von großem Interesse war auch, daß sich das $\mathbf{O p -}$ timụm der Milchsäurefällung bei den Rückenmarksflüssig- 
keiten bei Lues des Z.N.S. stets bei $0,3 \mathrm{ccm}$ Milchsäure fand. $\mathrm{Ob}$ sich hierin etwas für Lues Spezifisches erblicken läßt, müssen weitere Untersuchungen lehren.

\section{III.}

Es könnte nach den eben geschilderten Versuchen, die heterogenen Arbeitsgebieten entstammen, den Eindruck machen, als wären wir von der im Titel gegebenen Fragestellung abgewichen. In Wirklichkeit ist es aber nicht der Fall, denn bei den verschiedenen Ansichten und Ergebnissen, die über die Eiweißverhältnisse des Syphilitikerblutes bestehen, mußten wir, um die vorhandene Lücke, i. e. die Anwendung auf den Liquor, wenigstens teilweise auszufüllen, die verschiedenen Forschungsgebiete berücksichtigen. Dabei hat sich ergeben, daß bisher nur die WaR. und vielleicht die Flockungsreaktionen von SachsGeorgi und Mei nic ke im Liquor für Lues spezifisch sind. Eine für Lues spezifische Eiweißreaktion im Liquor kannten wir bisher nicht. Aus unseren Versuchen geht hervor, daß wir einer solchen vielleicht durch Versuche, die den physikalischen Chemismus betreffen (Milchsäureversuche nach Bruck) oder durch kolloidchemische Methoden (Kolloidschutzkurven!) am nächsten kommen werden, wenn das überhaupt möglich ist. Aber wir sehen einerseits, daß es absolut noch nicht feststeht, daß der WaR. bei Lues nur eine Veränderung irgendwelcher Eiweißkörper zugrunde liegen muß, wenn wir auch freilich noch keinen strikten Beweis dafür haben, daß Eiweißabbauprodukte die WaR. primär hervorrufen. Unsere Versuche hatten ja bezüglich beider Ansichten negative Ergebnisse. Andererseits ist es eine Frage, ob wir überhaupt im Liquor einen für Lues spezifischen Eiweißkörper oder eine derartige Eiweißreaktion werden nachweisen können, selbst wenn jener oder diese im Blute vorhanden sind. Zwar stammt im normalen Zustande das Eiweiß der Rückenmarksflüssigkeiten zum größten Teil aus dem Blute; dieser Übergang kann wohl im krankhaften Zustande vermehrt sein, das Eiweiß entstammt aber größtenteils den erkrankten Mengen selbst. Auf diesem Gebiete läßt sich aber nichts voraussagen und es ist nur zu hoffen, daß sich auf den von uns beschrittenen Wegen doch noch eine für Iues spezifische Reaktion und im Anschluß daran ein für Syphilis spezifischer Eiweißkörper im Liquor wird nachweisen lassen. Die Praxis würde davon Nutzen ziehen, speziell für jene nicht seltenen Fälle von Syphilis des Zentralnervensystems, bei dem die WaR. im Liquor (evtl. auch im Blute) negativ ist, vor allem aber auch die Theorie, indem dank Aufdeckung der syphilitischen Veränderungen der Körpersäfte viele schwebende Fragen der Syphilis ihre Beantwortung finden wïrden. 